\title{
Exact Supersymmetric Solution of Schrödinger Equation for central confining Potentials by using the Nikiforov-Uvarov Method
}

\author{
Metin Aktaş and Ramazan Sever* \\ Department of Physics, Middle East Technical University \\ 06531 Ankara, Turkey
}

November 10, 2018

\begin{abstract}
We present the exact supersymmetric solution of Schrödinger equation with the Morse, Pöschl-Teller and Hulthén potentials by using the Nikiforov-Uvarov method. Eigenfunctions and corresponding energy eigenvalues are calculated for the first six excited states. Results are in good agreement with the ones obtained before.
\end{abstract}

PACS numbers: 03.65.Ge, 12.39.Jh

Keywords: SUSYQM, Nikiforov-Uvarov Method, Morse Potential, Pöschl-Teller potential, Hulthén potential

\footnotetext{
${ }^{*}$ Corresponding author: sever@metu.edu.tr
} 


\section{Introduction}

Supersymmetric quantum mechanics (SUSYQM) is a framework used to determine energy eigenvalues and eigenfunctions of quantum mechanical problems. Various methods have been used in their solutions [1]. One of them is the factorization method introduced by Schrödinger to study the hydrogen atom problem [2]. This method was developed later by Infeld and Hull to categorize the analytically solvable potential problems [3]. Also it was used to derive in the solutions of certain non-linear equations [4]. The others are approximation methods known as $1 / N$ expansion [5], $\delta$-expansion [6], supersymmetric WKB (SWKB) [7, 8] and variational methods [9]. About two decades ago, Gendenshtein introduced a concept "shape invariance" to obtain the exact energy eigenvalues of the Schrödinger equation $[12,13]$. It provides us a simple approach to the solution when the potentials have shape invariance property. Recently, an alternative method known as the Nikiforov-Uvarov method (NU-method) has been introduced for solving the Schrödinger equation (SE). There have been several applications of SE with some well-known potentials [24, 25, 26], Dirac and Klein-Gordon equations for a Coulomb potential by using this method as well [27].

This article is organised as follows: In Sec. (2), we give a summary of SUSYQM. In Sec. (3), we introduce the NU-method to solve SE. In Sec. (4), solution of Morse, Pöschl-Teller and Hulthén potentials are obtained. Numerical results are given in the tables. Finally, we discuss the results in the conclusion.

\section{Summary of SUSYQM}

Supersymmetric algebra allows us to write Hamiltonians as [1]

$$
H_{ \pm}=-\frac{\hbar^{2}}{2 m} \frac{d^{2}}{d x^{2}}+V_{ \pm}(x)
$$

where The supersymmetric partner potentials $V_{ \pm}(x)$ in terms of the superpotential $W(x)$ are given by

$$
V_{ \pm}(x)=W^{2} \pm \frac{\hbar}{\sqrt{2 m}} \frac{d W}{d x} .
$$

The superpotential has a definition 


$$
W(x)=-\frac{\hbar}{\sqrt{2 m}}\left[\frac{d \ln \Psi_{0}^{(0)}(x)}{d x}\right],
$$

where, $\Psi_{0}^{(0)}(x)$ denotes the ground state wave function that satisfies the relation

$$
\Psi_{0}^{(0)}(x)=N_{0} \exp \left[-\frac{\sqrt{2 m}}{\hbar} \int^{x} W\left(x^{\prime}\right) d x^{\prime}\right] .
$$

The Hamiltonian $H_{ \pm}$can also be written in terms of the bosonic operators $A^{-}$and $A^{+}$

$$
H_{ \pm}=A^{\mp} A^{ \pm}
$$

where

$$
A^{ \pm}= \pm \frac{\hbar}{\sqrt{2 m}} \frac{d}{d x}+W(x)
$$

It is remarkable result that the energy eigenvalues of $H_{-}$and $H_{+}$are identical except for the ground state. In the case of unbroken supersymmetry, the ground state energy of the Hamiltonian $H_{-}$is zero $\left(E_{0}^{(0)}=0\right)$ [14]. In the factorization of the Hamiltonian, the Eqs. (1), (5) and (6) are used respectively. Hence, we obtain

$$
\begin{aligned}
H_{1}(x) & =-\frac{\hbar^{2}}{2 m} \frac{d^{2}}{d x^{2}}+V_{1}(x) \\
& =\left(A_{1}^{+} A_{1}^{-}\right)+E_{1}^{(0)}
\end{aligned}
$$

Comparing each side of the Eq. (7) term by term, we get the Riccati equation for the superpotential $W_{1}(x)$

$$
W_{1}^{2}-W_{1}^{\prime}=\frac{2 m}{\hbar^{2}}\left(V_{1}(x)-E_{1}^{(0)}\right) .
$$

Let us now construct the supersymmetric partner Hamiltonian $H_{2}$ as

$$
\begin{aligned}
H_{2}(x) & =-\frac{\hbar^{2}}{2 m} \frac{d^{2}}{d x^{2}}+V_{2}(x) \\
& =\left(A_{2}^{-} A_{2}^{+}\right)+E_{2}^{(0)}
\end{aligned}
$$

and Riccati equation takes

$$
W_{2}^{2}+W_{2}^{\prime}=\frac{2 m}{\hbar^{2}}\left(V_{2}(x)-E_{2}^{(0)}\right) .
$$


Similarly, one can write in general the Riccati equation and Hamiltonians by iteration as

$$
\begin{aligned}
W_{n}^{2} \pm W_{n}^{\prime} & =\frac{2 m}{\hbar^{2}}\left(V_{n}(x)-E_{n}^{(0)}\right) \\
& =\left(A_{n}^{ \pm} A_{n}^{\mp}\right)+E_{n}^{(0)},
\end{aligned}
$$

and

$$
\begin{aligned}
H_{n}(x) & =-\frac{\hbar^{2}}{2 m} \frac{d^{2}}{d x^{2}}+V_{n}(x) \\
& =A_{n}^{+} A_{n}^{-}+E_{n}^{(0)}, \quad n=1,2,3, \ldots
\end{aligned}
$$

where

$$
A_{n}^{ \pm}= \pm \frac{\hbar}{\sqrt{2 m}} \frac{d}{d x}+\frac{d}{d x}\left(\ln \Psi_{n}^{(0)}(x)\right) .
$$

Because of the SUSY unbroken case, the partner Hamiltonians satisfy the following expressions $[9,14]$

$$
E_{n+1}^{(0)}=E_{n}^{(1)}, \quad \text { with } \quad E_{0}^{(0)}=0, \quad n=0,1,2, \ldots
$$

and also the wave function with the same eigenvalue can be written as [14]

$$
\Psi_{n}^{(1)}=\frac{A^{-} \Psi_{n+1}^{(0)}}{\sqrt{E_{n}^{(0)}}}
$$

with

$$
\Psi_{n+1}^{(0)}=\frac{A^{+} \Psi_{n}^{(1)}}{\sqrt{E_{n}^{(0)}}} .
$$

This procedure is known as the hierarchy of Hamiltonians.

\section{The Nikiforov-Uvarov Method}

The NU-method reduces the second order differential equations (ODEs) to the hypergeometric type with an appropriate coordinate transformation $x=x(s)$ as

$$
\Psi^{\prime \prime}(s)+\frac{\tilde{\tau}(s)}{\sigma(s)} \Psi^{\prime}(s)+\frac{\tilde{\sigma}(s)}{\sigma^{2}(s)} \Psi(s)=0
$$


where $\sigma(s)$ and $\tilde{\sigma}(s)$ are polynomials with at most second degree, and $\tilde{\tau}(s)$ is a polynomial with at most first degree $[24,25,26,27]$. If we take the following factorization

$$
\Psi(s)=\phi(s) y(s)
$$

the Eq. (17) becomes [27]

$$
\sigma(s) y^{\prime \prime}(s)+\tau(s) y^{\prime}(s)+\Lambda y(s)=0
$$

where

$$
\sigma(s)=\pi(s) \frac{d}{d s}(\ln \phi(s))
$$

and

$$
\tau(s)=\tilde{\tau}(s)+2 \pi(s) .
$$

Also, $\Lambda$ is defined as

$$
\Lambda_{n}+n \tau^{\prime}+\frac{\left[n(n-1) \sigma^{\prime \prime}\right]}{2}=0, \quad n=0,1,2, \ldots
$$

The energy eigenvalues can be calculated from the above equation. We first have to determine $\pi(s)$ and $\Lambda$ by defining

$$
k=\Lambda-\pi^{\prime}(s)
$$

Solving the quadratic equation for $\pi(s)$ with the Eq. (23), we get

$$
\pi(s)=\left(\frac{\sigma^{\prime}-\tilde{\tau}}{2}\right) \pm \sqrt{\left(\frac{\sigma^{\prime}-\tilde{\tau}}{2}\right)^{2}-\tilde{\sigma}+k \sigma} .
$$

Here, $\pi(s)$ is a polynomial with the parameter $s$ and prime factors denote the differentials at first degree. The determination of $k$ is the essential point in the calculation of $\pi(s)$. It is simply defined as by setting the discriminant of the square root must be zero [27]. Therefore, we obtain a general quadratic equation for $k$.

The determination of the wave function is now in order. We consider the Eq. (20) and the Rodrigues relation

$$
y_{n}(s)=\frac{C_{n}}{\rho(s)} \frac{d^{n}}{d s^{n}}\left[\sigma^{n}(s) \rho(s)\right]
$$


where $C_{n}$ is normalizable constant and the weight function $\rho(s)$ satisfy the following relation

$$
\frac{d}{d s}[\sigma(s) \rho(s)]=\tau(s) \rho(s)
$$

The Eq. (25) refers to the classical orthogonal polynomials that have many important properties especially orthogonality relation can be defined as

$$
\int_{a}^{b} y_{n}(s) y_{m}(s) \rho(s) d s=0, \quad m \neq n
$$

\section{Calculations}

We will use the Nikiforov-Uvarov method by getting the hypergeometric or confluent hypergeometric form of the Schrödinger equation with the following potentials for $s-$ states only.

\subsection{Morse Potential}

The Morse potential is

$$
V_{M}(r)=D\left(e^{-2 a x}-2 e^{-a x}\right),
$$

with $x=\left(r-r_{0}\right) / r_{0}[23]$. Here, $\mathrm{D}$ denotes the dissociation energy parameter and $r_{0}$ is the equilibrium distance between nucleus. Its supersymmetric form becomes [1]

$$
W(x)=A-B e^{-a x} .
$$

Thus, we first get the superpartner potentials as

$$
V_{ \pm}(x, A, B)=A^{2}+B^{2} e^{-2 a x}-2 B\left(A \mp \frac{\hbar a}{\sqrt{8 m}}\right) e^{-a x} .
$$

From the Eq. (1), Schrödinger equation can be written as [8]

$$
H_{ \pm} \Psi=E_{ \pm} \Psi
$$

or explicitly

$$
\frac{d^{2} \Psi}{d x^{2}}+\frac{2 m}{\hbar^{2}}\left[E_{-}-V_{-}\right] \Psi=0
$$


Using the Eq. (30) for $V_{-}$, we get

$$
\Psi^{\prime \prime}(x)+\frac{2 m}{\hbar^{2}}\left[\bar{E}-\bar{B} e^{-2 a x}+\bar{C} e^{-a x}\right] \Psi(x)=0,
$$

where $\bar{E}=E_{-}-A^{2}, \bar{B}=B^{2}$ and $\bar{C}=2 B\left(A+\frac{\hbar a}{\sqrt{8 m}}\right)$. By introducing a transformation

$$
-s=e^{-a x}
$$

the Eq. (33) takes the form

$$
\Psi^{\prime \prime}(s)+\frac{1}{s} \Psi^{\prime}(s)+\frac{1}{s^{2}}\left[\beta^{2}-\gamma^{2} s-\alpha^{2} s^{2}\right] \Psi(s)=0
$$

where

$$
\alpha^{2}=\frac{2 m \bar{B}}{a^{2} \hbar^{2}}, \quad \beta^{2}=\frac{2 m \bar{E}}{a^{2} \hbar^{2}}\left(E^{\prime}<0\right) \quad \text { and } \quad \gamma^{2}=\frac{2 m \bar{C}}{a^{2} \hbar^{2}} .
$$

Comparing the Eq. (35) with the Eq. (17), we obtain

$$
\sigma(s)=s, \quad \tilde{\tau}(s)=1 \quad \text { and } \quad \tilde{\sigma}(s)=\left(-\beta^{2}-\gamma^{2} s-\alpha^{2} s^{2}\right)
$$

Substituting these polynomials into the Eq. (24), we get

$$
\pi(s)= \pm \sqrt{\alpha^{2} s^{2}+\left(k+\gamma^{2}\right) s+\beta^{2}} .
$$

The constant $k$ is determined as

$$
k_{1,2}=-\gamma^{2} \pm 2 \alpha \beta
$$

and we have

$$
\pi(s)= \begin{cases} \pm(\alpha s-\beta), & \text { for } \quad k=-\gamma^{2}-2 \alpha \beta \\ \pm(\alpha s+\beta), & \text { for } \quad k=-\gamma^{2}+2 \alpha \beta .\end{cases}
$$

A proper value for $\pi(s)$ is chosen, so that the function

$$
\tau(s)=(1+2 \beta)-2 \alpha s,
$$


has a negative derivative [27]. By using the Eq. (22), we can find

$$
\begin{aligned}
\Lambda & =-\gamma^{2}-2 \alpha \beta-\alpha \\
& =2 \alpha n .
\end{aligned}
$$

Thus, we simply get the energy eigenvalues as

$$
\bar{E}_{n, \ell=0}=\frac{a^{2} \hbar^{2}}{2 m}\left[\bar{D}-\left(n+\frac{1}{2}\right)\right]^{2}
$$

where $\bar{D}=\gamma^{2} / 2 \alpha$. By setting $\alpha=1$, this equation reduces to the Eq. (10) as [28] for $s$ - states. The Eq. (43) can be seperable. Its square term refers to the anharmonic oscillator correction and the other one corresponds to the harmonic oscillator solution.

Now, we are going to determine the eigenfunctions for this potential. By considering the Eq. (18) and using the Eq. (20), we obtain

$$
\phi(s)=s^{\beta} e^{-\alpha s} .
$$

By using the Eqs. (26) and (25), we obtain

$$
y_{n}(s)=\frac{C_{n}}{\rho(s)} \frac{d^{n}}{d s^{n}}\left[s^{n} \rho(s)\right]
$$

where $\rho(s)=s^{2 \beta} e^{-2 \alpha s}$. The Eq. (45) stands for the associated Laguerre polynomials, that is

$$
y_{n}(s) \equiv L_{n}^{t}(s)
$$

where $t=2 \beta$. Hence, we can write the wave function in the final form

$$
\Psi_{n}(x)=C_{n} s^{\beta} e^{-\eta s} L_{n}^{t}(s)
$$

with $s=-e^{a x}$. It is normalizable. Using the Eq. (27), the normalization constant can be found as

$$
C_{n}=\sqrt{\frac{n !}{\left(n+\beta+\frac{1}{2}\right)(n+2 \beta) !}}, \quad n=0,1,2 \ldots
$$




\subsection{Pöschl-Teller Potential}

The Pöschl-Teller potential is

$$
V_{P T}(x)=-\frac{U_{0}}{\cosh ^{2} \alpha x}
$$

where $U_{0}=\lambda(\lambda-1)>0$ [8]. Also, its superpotential potential is [1]

$$
W(x)=A \tanh \alpha x
$$

From Eq. (2), we get its superpartners

$$
V_{ \pm}(x, A)=A^{2}-A\left(A \mp \frac{\alpha \hbar}{\sqrt{2 m}}\right) \frac{1}{\cosh ^{2} \alpha x} .
$$

Thus, we can write the Schrödinger equation as

$$
\Psi^{\prime \prime}(x)+\frac{2 m}{\hbar^{2}}\left[\widetilde{E}-\frac{\kappa}{\cosh ^{2} \alpha x}\right] \Psi(x)=0,
$$

where $\widetilde{E}=E_{-}-A^{2}$ and $\kappa=A\left(A+\frac{\alpha \hbar}{\sqrt{2 m}}\right)$. Introducing a transformation

$$
s=\tanh \alpha x
$$

we rewrite the Eq. (52)

$$
\Psi^{\prime \prime}(s)+\frac{-2 s}{\left(1-s^{2}\right)} \Psi^{\prime}(s)+\frac{1}{\left(1-s^{2}\right)^{2}}\left[-\beta^{2}+\gamma^{2}\left(1-s^{2}\right)\right] \Psi(s)=0
$$

where

$$
\beta^{2}=\frac{2 m \widetilde{E}}{\alpha^{2} \hbar^{2}}(\widetilde{E}<0) \quad \text { and } \quad \gamma^{2}=\frac{2 m \kappa}{\alpha^{2} \hbar^{2}} .
$$

By comparing the Eq. (54) with the Eq. (17), we determine polynomials as

$$
\sigma(s)=\left(1-s^{2}\right), \quad \tilde{\tau}(s)=-2 s \quad \text { and } \quad \tilde{\sigma}(s)=-\beta^{2}+\gamma^{2}\left(1-s^{2}\right)
$$

Substituting them into the Eq. (24), we obtain

$$
\pi(s)= \pm \sqrt{\beta^{2}-\gamma^{2}\left(1-s^{2}\right)+k\left(1-s^{2}\right)}
$$


The constant $k$ is determined in the same way. Therefore, we get

$$
\pi(s)=\left\{\begin{array}{lll} 
\pm \beta, & \text { for } & k=\gamma^{2} \\
\pm \beta s, & \text { for } & k=\gamma^{2}-\beta^{2} .
\end{array}\right.
$$

Here we choose the proper value, so that

$$
\tau(s)=-2(1+\beta) s,
$$

has a negative derivative. From the Eq. (22), we calculate

$$
\begin{aligned}
\Lambda & =\gamma^{2}-\beta^{2}-\beta \\
& =n^{2}+n+2 n \beta .
\end{aligned}
$$

Hence, the energy eigenvalues are found as

$$
\widetilde{E}_{n}=A^{2}-\frac{\hbar^{2} \alpha^{2}}{2 m}\left[-\left(n+\frac{1}{2}\right)+\frac{1}{2} \sqrt{1+4 \gamma^{2}}\right]^{2} .
$$

The wave function $\Psi_{n}(x)$ is obtained from the Eq. (18) by taking $\pi(s)=-\beta s$ as follows. We first get

$$
\phi(s)=\left(1-s^{2}\right)^{\beta / 2},
$$

and using the Eqs. (26) and (25), we find

$$
y_{n}(s)=\frac{C_{n}}{\rho(s)} \frac{d^{n}}{d s^{n}}\left[\left(1-s^{2}\right)^{n} \rho(s)\right],
$$

where $\rho(s)=\left(1-s^{2}\right)^{\beta}$. The Eq. (63) stands for the Jacobi polynomials as

$$
y_{n} \equiv P_{n}^{(\beta, \beta)}(s)
$$

Hence, $\Psi_{n}(x)$ can be written in the following form

$$
\Psi_{n}(x)=C_{n}\left(1-s^{2}\right)^{\beta / 2} P_{n}^{(\beta, \beta)}(s),
$$

with $s=\tanh \alpha x$. Considering the Eq. (27), the normalization constant is obtained as

$$
C_{n}=\frac{1}{2^{\beta}(n+\beta) !} \sqrt{\frac{(2 n+2 \beta+1)}{2} n !(2 n+\beta) !} .
$$

where $n, \beta \geq 0$. 


\subsection{Hulthén Potential}

This potential can be solved exactly for $s$-states only. This is due to the similarity between Coulomb and Hulthén potentials. It plays an important role in the applications of quantum scattering theory. The Hulthén potential is given by [23]

$$
V_{0}^{H}=-V_{0} \frac{e^{-\lambda x}}{\left(1-e^{-\delta x}\right)},
$$

where $\delta=1 / a$, is the screening parameter. We get the supersymmetric form the potential for $s$-states [10]

$$
W_{1}=\bar{a}+\bar{b} \frac{e^{-\delta x}}{\left(1-e^{-\delta x}\right)} .
$$

Here, $\bar{a}$ and $\bar{b}$ are arbitrary constants. We can also write the supersymmetric partner of the potential as [11]

$$
V_{1}^{H}=V_{0}^{H}+\frac{V_{0}^{2} e^{-\delta x}}{\left(1-e^{-\delta x}\right)^{2}} .
$$

The second term in Eq. (69) behaves like centrifugal barrier [23]. The SE has the form

$$
\Psi^{\prime \prime}(x)+\frac{2 m}{\hbar^{2}}\left[\bar{E}-V_{1}^{H}(x)\right] \Psi(x)=0 .
$$

Using the transformation

$$
s=e^{-\delta x}
$$

we rewrite

$$
\Psi^{\prime \prime}(s)+\frac{(1-s)}{[s(1-s)]} \Psi^{\prime}(s)+\frac{1}{[s(1-s)]^{2}}\left[-\left(\varepsilon^{2}+\beta^{2}\right) s^{2}+\left(2 \varepsilon^{2}+\beta^{2}-\gamma^{2}\right) s-\varepsilon^{2}\right] \Psi(s)=0
$$

where

$$
\varepsilon^{2}=\frac{2 m \bar{E}}{\delta^{2} \hbar^{2}}(\bar{E}>0), \quad \beta^{2}=\frac{2 m V_{0}}{\delta^{2} \hbar^{2}} \quad \text { and } \quad \gamma^{2}=\frac{2 m V_{0}^{2}}{\delta^{2} \hbar^{2}}
$$

By comparing the Eq. (72) with the Eq. (17), we get

$$
\sigma(s)=s(1-s), \quad \tilde{\tau}(s)=1-s \quad \text { and } \quad \tilde{\sigma}(s)=-\left(\varepsilon^{2}+\beta^{2}\right) s^{2}+\left(2 \varepsilon^{2}+\beta^{2}-\gamma^{2}\right) s-\varepsilon^{2} .
$$


Substituting them into the Eq. (24), we obtain

$$
\pi(s)=-\frac{1}{2} s \pm \frac{1}{2} \sqrt{4\left(\varepsilon^{2}+\beta^{2}-k+\frac{1}{4}\right) s^{2}-4\left(2 \varepsilon^{2}+\beta^{2}-\gamma^{2}-k\right) s+4 \varepsilon^{2}} .
$$

From Eq. (75) $k$ is determined as

$$
k_{1,2}=-\gamma^{2}+\beta^{2} \pm \varepsilon \sqrt{1+4 \gamma^{2}} .
$$

Following the same procedure, we get

$$
\pi(s)=-\frac{1}{2} s \pm \frac{1}{2}\left[\left(2 \varepsilon+\sqrt{1+4 \gamma^{2}}\right) s-2 \varepsilon\right],
$$

and the energy eigenvalues for the supersymmetric Hulthén potential becomes

$$
\bar{E}_{\bar{n}, \ell=0}=-V_{0}\left[\frac{\beta^{2}-\bar{n}^{2}}{2 \bar{n} \beta}\right]^{2}, \quad \bar{n}=1,2, \ldots
$$

Here, $\beta^{2}=2 V_{0} / \delta^{2}$ with $(\hbar=m=1)$ and $\bar{n}=\left[\left(n+\frac{1}{2}\right)-\frac{1}{2} \sqrt{1+4 \gamma^{2}}\right]$. If the limit $\gamma \rightarrow 0$ is chosen, the energy eigenvalue reduces to the form obtained from the usual solution of the Hulthén potential.

The wave functions can now be obtained similarly from the Eq. (18). Using the Eq. (20), we have

$$
\phi(s)=s^{\varepsilon}(1-s)^{\mu / 2},
$$

where $\mu=1+\sqrt{1+4 \gamma^{2}}$. The Eqs. (26) and (25) lead to

$$
y_{n}(s)=\frac{C_{n}}{\rho(s)} \frac{d^{n}}{d s^{n}}\left[s^{n}(1-s)^{n} \rho(s)\right] .
$$

Here, $\rho(s)=s^{2 \varepsilon}(1-s)^{\mu-1}$. It stands for the Jacobi polynomials as [25]

$$
y_{n}(s) \simeq P_{n}^{(2 \varepsilon, \mu-1)}(1-2 s)
$$

Thus, the final form of the wave function can also be written in terms of the Jacobi polynomials resulting

$$
\Psi_{n}(x)=C_{n} s^{\varepsilon}(1-s)^{\mu / 2} P_{n}^{(2 \varepsilon, \mu-1)}(1-2 s),
$$


with $s=e^{-\delta x}$, and also the normalization constant $C_{n}$.

\section{Conclusions}

We have obtained the exact supersymmetric solution of some central confining potentials by applying the Nikiforov-Uvarov Method. The eigenfunctions and corresponding energy egenvalues of the these three well-known shape invariant potentials, i.e. Morse, PöschlTeller and Hulthén are calculated analytically. All the wave functions are physical. We present numerical results in tabular form for $\ell=0$. In Table I, we list energy eigenvalues of $H_{2}$ molecule by taking $D=4.7446 \mathrm{eV}, a=1.9425 \AA^{-1}$ and $m=0.50391 \mathrm{amu}$ for ground state and first eleven excited states. The energy difference between the successive states decreases. In Table II, the six excited energy states of the the potential are given for various values of $n$ and $\lambda$ with $A=(1+\sqrt{1+4 \lambda(\lambda-1)}) / 2$. In Table III, the first five excited energy levels are tabulated for $s$-states with different values of screening parameter $\delta$. Our results are in good agreement with the ones obtained by the other methods. 


\section{References}

[1] F. Cooper, A. Khare, U. Sukhatme, Phys. Rep., 251 (1995) 267

[2] E. Schrödinger, Proc. R. Irish Acad., 46A (1940) 183

[3] L. Infeld, T. E. Hull, Rev. Mod. Phys., 23 (1951) 21

[4] V. B. Matveev, Lett. Math. Phys., 3 (1979) 217

[5] T. Imbo, U. Sukhatme, Phys. Rev. Lett. 54 (1985) 2184

[6] F. Cooper, P. Roy, Phys. Lett. A 143 (1990) 202

[7] A. Comtet, A. Bandrauk, D. K. Campbell, Phys. Lett. B 150 (1985) 159

[8] E. Kasap, B. Gönül, M. Şimşek, Chem. Phys. Lett., 172 (1990) 499

[9] E. D. Filho, R. M. Ricotta, Phys. Lett. A, 269 (2000) 269

[10] B. Gönül, O. Özer, Y. Cançelik, M. Koçak, Phys. Lett. A, 275 (2000) 238

[11] U. Laha, C. Bhattacharyya, K. Roy, B. Talukdar, Phys. Rev. C, 38, (1988) 558

[12] L. E. Gendenstein, I. V. Krive, Usp. Fiz. Nauk 146 (1985) 553

[13] L. E. Gendenstein, JETP Lett. 38 (1983) 356

[14] F. Cooper, J. N. Ginocchio, A. Khare, Phys. Rev. D, 36 (1987) 2458

[15] R. Dutt, A. Khare, U. Sukhatme, Am. J. Phys. A, 56 (1988) 163

[16] G. Levai, J. Phys. A, 22 (1989) 267

[17] J. W. Dabrowska, A. Khare, U. Sukhatme, J. Phys. A, 21 (1998) L195

[18] D. T. Barclay, R. Dutt, A. Gangopadhyaya, A. Khare, A. Pagnamenta, U. Sukhatme, Phys. Rev. A, 48 (1993) 2786

[19] F. Cooper, A. Khare, J. Phys. A, 26 (1993) L901

[20] A. Khare, U. Sukhatme, J. Phys. A, 22 (1989) 2847

[21] B. Talukdar, U. Das, C. Bhattacharyaya, P. K. Bera, J. Phys. A, 25 (1992) 4073 
[22] C. V. Sukumar, J. Phys. A, 18 (1985) 2917

[23] S. Flügge, "Practical Quantum Mechanics I" (Springer-Verlag, New York, (1974)

[24] H. Eğrifes, D. Demirhan, F. Büyükkılıç, Physica Scripta, 59 (1999) 90

[25] H. Eğrifes, D. Demirhan, F. Büyükkılıç, Physica Scripta, 60 (1999) 195

[26] F. Büyükkılıç, H. Eğrifes, D. Demirhan, Theor. Chem. Acc., 98 (1997) 192

[27] A. F. Nikiforov, V. B. Uvarov, "Special Functions of Mathematical Physics" (Birkhauser, Basel, 1988)

[28] D. A. Morales, Chem. Phys. Lett., 161 (1989) 253

[29] M. Bag, M. M. Panja, R. Dutt, Y. P. Varshni, 222 (1994) 46

[30] A. Z. Tang, F. T. Chan Phys. Rev. A, 35 (1987) 911 
Table I: Vibrational (non-rotating) energy eigenvalues of the Morse potential for $H_{2}$ molecule.

\begin{tabular}{cccc}
\hline \hline Quantum no $(\mathbf{n})$ & Eigenvalues $[\mathbf{2 8}]$ & $\overline{\mathbf{E}}_{\mathbf{n}}($ Our work $)$ & $\overline{\mathbf{E}}_{\mathbf{n}+\mathbf{1}}-\overline{\mathbf{E}}_{\mathbf{n}}$ \\
\hline 0 & -4.47600 & -4.47610 & 0.51354 \\
\hline 1 & & -3.96256 & 0.48226 \\
2 & & -3.48030 & 0.45100 \\
3 & -2.22050 & -3.02930 & 0.41968 \\
4 & -1.86330 & -2.60962 & 0.38842 \\
\hline 5 & -1.53740 & -2.22120 & 0.35713 \\
6 & & -1.86407 & 0.32587 \\
7 & & -1.53820 & 0.29456 \\
\hline 8 & & -1.24364 & 0.26330 \\
9 & & -0.98034 & 0.23201 \\
10 & & -0.74833 & 0.20073 \\
\hline
\end{tabular}


Table II: Eigenvalues of the Pöschl-Teller potential with $A=(1+\sqrt{1+4 \lambda(\lambda-1)}) / 2$ setting by $\left(\hbar=\alpha=2 m=1, A^{2}=0\right.$ and $\left.\kappa=\gamma^{2}\right)$ in Eq. (61) [29].

\begin{tabular}{|c|c|c|c|c|c|c|}
\hline$\lambda$ & $-\widetilde{\mathbf{E}}_{1}$ & $-\widetilde{\mathbf{E}}_{2}$ & $-\widetilde{\mathbf{E}}_{3}$ & $-\widetilde{\mathbf{E}}_{4}$ & $-\widetilde{\mathbf{E}}_{5}$ & $-\widetilde{\mathbf{E}}_{6}$ \\
\hline 2.0 & 0.0 & 1.0 & 4.0 & 9.0 & 16.0 & 25.0 \\
\hline 6.0 & & 0.0 & 1.0 & 4.0 & 9.0 & 16.0 \\
\hline 12.0 & & & 0.0 & 1.0 & 4.0 & 9.0 \\
\hline 20.0 & & & & 0.0 & 1.0 & 4.0 \\
\hline 30.0 & & & & & 0.0 & 1.0 \\
\hline 42.0 & & & & & & 0.0 \\
\hline
\end{tabular}


Table III: Eigenvalues of the Hulthén potential for several values of screening parameter $\delta$, substituting $V_{0}=\delta / 4$ in $\beta^{2}$ of the Eq. (78).

\begin{tabular}{|c|c|c|c|}
\hline Quantum no (n) & $-\mathbf{E}_{\mathbf{n}}[30]$ & $-\mathbf{E}_{\text {exact }}$ & $-\overline{\mathbf{E}}_{\mathbf{n}}($ Our work $)$ \\
\hline \multicolumn{4}{|c|}{$\delta=0.002$} \\
\hline 1 & 0.4990005 & 0.4990005 & 0.4990005 \\
\hline 2 & 0.1240020 & 0.1240020 & 0.1240020 \\
\hline 3 & 0.0545601 & 0.0545601 & 0.0545601 \\
\hline 4 & 0.0302580 & 0.0302580 & 0.0302580 \\
\hline 5 & & & 0.0012500 \\
\hline \multicolumn{4}{|c|}{$\delta=0.01$} \\
\hline 1 & 0.4950125 & 0.4950125 & 0.4950125 \\
\hline 2 & 0.1200500 & 0.1200500 & 0.1200500 \\
\hline 3 & 0.0506681 & 0.0506681 & 0.0506681 \\
\hline 4 & 0.0264501 & 0.0264500 & 0.0264500 \\
\hline 5 & 0.0153128 & 0.0153125 & 0.0153125 \\
\hline \multicolumn{4}{|c|}{$\delta=0.05$} \\
\hline 1 & 0.4753125 & 0.4753125 & 0.4753125 \\
\hline 2 & 0.1012503 & 0.1012500 & 0.1012500 \\
\hline 3 & 0.0333746 & 0.0333681 & 0.0333681 \\
\hline 4 & 0.0113035 & 0.0112500 & 0.0112500 \\
\hline 5 & & & 0.0028125 \\
\hline \multicolumn{4}{|c|}{$\delta=0.02$} \\
\hline 1 & & & 0.4900500 \\
\hline 2 & & & 0.1152000 \\
\hline 3 & 0.0460057 & 0.0460056 & 0.0460056 \\
\hline 4 & 0.0220512 & 0.0220500 & 0.0220500 \\
\hline 5 & 0.0112554 & 0.0112500 & 0.0112550 \\
\hline \multicolumn{4}{|c|}{$\delta=0.2$} \\
\hline 1 & 0.4049962 & 0.4050000 & 0.4050000 \\
\hline 2 & 0.0450856 & 0.0450000 & 0.0450000 \\
\hline 3 & & & 0.0005556 \\
\hline 4 & & & 0.0112500 \\
\hline 5 & & & 0.0450000 \\
\hline
\end{tabular}

